\title{
Deterministic transfer of spin polarization in wire-like lateral structures via the persistent spin helix
}

\author{
M. Schwemmer, A. Hanninger, M. Weingartner, M. Oltscher, M. Ciorga, D. Weiss, D. Schuh, \\ D. Bougeard, T. Korn, and C. Schüller ${ }^{\text {a) }}$ \\ Institut für Experimentelle und Angewandte Physik, Universität Regensburg, D-93040 Regensburg, Germany
}

(Received 14 September 2016; accepted 13 October 2016; published online 27 October 2016)

\begin{abstract}
We used spatially- and time-resolved Kerr rotation microscopy to show that in lateral wire-like structures, based on a modulation-doped GaAs-AlGaAs quantum well, an optically initialized spin polarization can be deterministically transferred to specific lateral positions, employing the persistent spin helix (PSH). To this end, we show that confinement in two directions leads to a strong enhancement of the effective decay time of spin polarization, which can be exploited to transfer spin polarization over relatively large lateral distances. This is demonstrated by the investigation of L-shaped wire-like lateral structures, where the legs are positioned in directions parallel and perpendicular to the wave vector of the PSH. Published by AIP Publishing.

[http://dx.doi.org/10.1063/1.4966184]
\end{abstract}

The key tool to manipulate the spin in semiconductorbased devices is spin-orbit interaction. ${ }^{1}$ However, on the downside, the spin-orbit interaction leads to spin dephasing, which limits the performance of spintronics or spin-based information processing devices. In this respect, an important milestone has been the proposal of a $\mathrm{SU}(2)$ spin rotation symmetry in quantum wells, based on the zincblende-type semiconductors, by Bernevig et $\mathrm{al}^{2}$ For (001)-grown quantum wells, this outstanding situation occurs for balanced linear Rashba ${ }^{3}$ and Dresselhaus ${ }^{4}$ spin-orbit fields. Very appealingly, the $\mathrm{SU}(2)$ symmetry ideally protects the system against Dyakonov-Perel spin dephasing 5 and should support a helical mode, the so called persistent spin helix (PSH), which opens up interesting application possibilities. Even before the proposal of Bernevig et al., Schliemann et al. suggested a non-ballistic spin field-effect transistor, based on the same theoretical principles of balanced spin-orbit fields. ${ }^{6}$ Since then, a wealth of experimental and theoretical investigations has appeared along these lines. Most prominent are the first experimental evidences of the existence of a PSH by transient spin grating spectroscopy, ${ }^{7}$ direct mapping via time- and spatially-resolved Kerr microscopy, ${ }^{8}$ and weak localization/antilocalization experiments. ${ }^{9}$ Closely related, a spin Hall effect transistor was demonstrated by Wunderlich et al. ${ }^{10}$ Subsequently, the PSH was investigated in, e.g., gated structures, ${ }^{11}$ laterally confined structures, ${ }^{12}$ structures with imbalanced spin-orbit interactions ${ }^{13,14}$ or via inelastic light scattering. ${ }^{15}$ Very recently, investigations in biased structures under charge transport were reported. ${ }^{16,17}$

In this work, we used spatially- and time-resolved Kerr rotation microscopy to show that spin polarization can be deterministically transferred to specific lateral positions via the PSH. We started by experimentally verifying the PSH in the as-grown sample, a GaAs-AlGaAs single quantum well, via spatially-resolved Kerr microscopy, followed by the investigation of narrow channels with lateral widths down to $3 \mu \mathrm{m}$. These experiments have shown that the effective

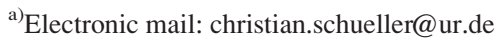

decay time of the spin polarization is prolonged by a factor of about three in narrow channels, as compared to the twodimensional (2D) case. Finally, we used L-shaped lateral structures to transfer spin polarization to specific lateral positions, employing the two distinct inplane directions, parallel and perpendicular to the wave vector of the PSH.

The sample contains a (001)-grown, one-side nmodulation-doped $\mathrm{GaAs}-\mathrm{Al}_{0.3} \mathrm{Ga}_{0.7} \mathrm{As}$ single quantum well, hosting a two-dimensional electron system (2DES) with electron density $n \sim 5.9 \times 10^{11} \mathrm{~cm}^{-2}$ and mobility $\mu \sim 8.4 \times 10^{5}$ $\mathrm{cm}^{2} \mathrm{~V}^{-1} \mathrm{~s}^{-1}$, measured via magneto transport under illumination at $T=4.2 \mathrm{~K}$. Figure 1 (a) shows a schematic picture of the conduction-band profile of the active region of the sample. It consists of a $12 \mathrm{~nm}$ GaAs quantum well with a Si deltadoping layer, separated by a $20 \mathrm{~nm}$-thick AlGaAs spacer. The sample parameters are designed for equal magnitudes of the linear Rashba and Dresselhaus coefficients, $\alpha$ and $\beta$, respectively. ${ }^{2}$ The electric field, due to space charges, inside the quantum well is pointing in $[00 \overline{1}]$ direction [cf. Fig. 1(a)]. This means that for our sample $\alpha=-\beta$, with $\beta$ being positive. ${ }^{18}$ The spin-orbit Hamiltonian of an electron in a (001)oriented quantum well is given by $H_{\mathrm{SO}}=\alpha\left(k_{010} \sigma_{x}-k_{100} \sigma_{y}\right)$ $+\beta\left(k_{100} \sigma_{x}-k_{010} \sigma_{y}\right)$, if terms linear in $\mathbf{k}_{\|}=\left(k_{100}, k_{010}\right)$ are considered, only. The effective $\mathbf{k}_{\|}$-dependent spin-orbit field $\mathbf{B}_{\text {eff }}$ is usually defined via a Zeeman-type Hamiltonian $H_{\mathrm{SO}}=\hat{\sigma} \mathbf{B}_{\text {eff }}$, where $\hat{\sigma}=\left(\sigma_{x}, \sigma_{y}, \sigma_{z}\right)$ contains the Pauli spin matrices. For $\alpha=-\beta$, this results in an effective spin-orbit field $\mathbf{B}_{\text {eff }}\left(\mathbf{k}_{\|}\right)=\beta\left(k_{100}-k_{010}\right) \mathbf{k}_{110} / k_{110}$ [red arrows in Fig. 1(a)], which is for all $\mathbf{k}_{\|}$either parallel or antiparallel to the [110] inplane direction of the quantum well, depending on the sign of the sum $\left(k_{100}-k_{010}\right)$. With these parameters, the PSH is supported in the [1 10$]$ direction for spins that are initially aligned perpendicular to the quantum-well plane. ${ }^{2}$ For simplicity, we assign for the following considerations the [110] and [110] directions as $x$ and $y$, respectively [see inset between Figs. 1(c) and 1(d)], so that the $x$ direction is the direction of the wave vector of the PSH.

The experiments are performed with a mode-locked Ti:Sapphire laser, operated at a central wavelength of $795 \mathrm{~nm}$ 
(a)

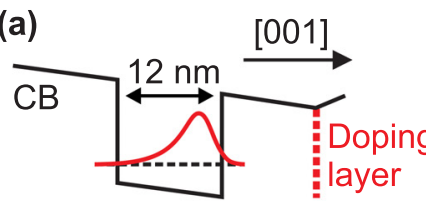

AlGaAs GaAs AIGaAs
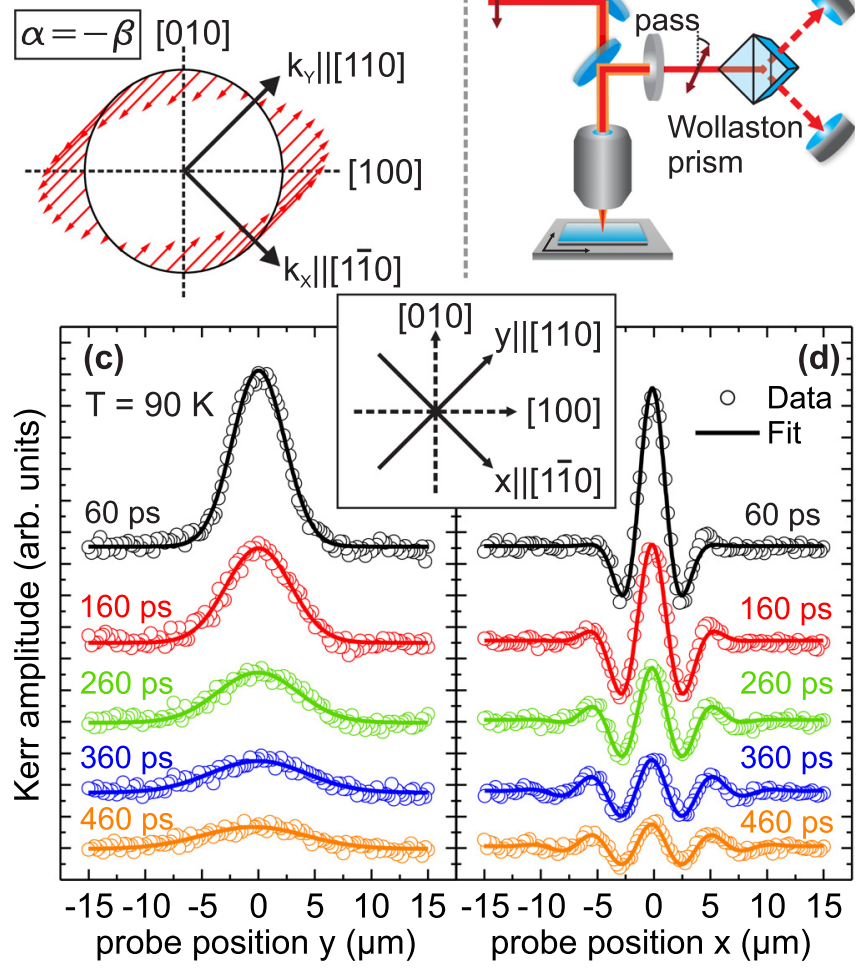

FIG. 1. (a) Schematic picture of the conduction band-edge profile of the sample and of the effective spin-orbit field $\mathbf{B}_{\text {eff }}$ (red arrows) at the Fermi energy in $k$ space. (b) Setup for spatially and time-resolved Kerr rotation microscopy. (c) Line scans of the Kerr rotation signal along the $y$ direction (see inset) for different time delays $\Delta t$. The pump spot is fixed at $y=0$. (d) Same as (c) but along the $x$ direction. The pump spot is fixed at $x=0$.

and a repetition rate of $80 \mathrm{MHz}$. The experimental setup is sketched in Fig. 1(b). The laser beam is divided into two pulse trains (pump and probe beams) via a beam splitter, and a time delay $\Delta t$ between pump and probe pulses can be adjusted via a mechanical delay line. The individual pulses have a pulse duration of about $0.1 \mathrm{ps}$, and a spectral full width at half maximum (FWHM) of about $12 \mathrm{~nm}$. Via optical band-pass filters, the pump pulses are spectrally shaped with a center wavelength of $780 \mathrm{~nm}$ and FWHM of about $10 \mathrm{~nm}$ from the highenergy tails of the plain pulses, and probe pulses with a center wavelength of $810 \mathrm{~nm}$ and FWHM of about $10 \mathrm{~nm}$ from the low-energy tails of the plain pulses [orange and red beams in Fig. 1(b)]. After all optical components, in particular, the band-pass filters, the pulse duration is about $0.4 \mathrm{ps}$, with no significant chirp of the pulses. With these settings, the energy of the pump pulses is at the onset of the absorptive region, and the energy of the probe pulses in the transparent region of the quantum well. ${ }^{19}$ The used power densities are on the order of $40 \mathrm{~kW} / \mathrm{cm}^{2}\left(90 \mathrm{~kW} / \mathrm{cm}^{2}\right)$ for the pump (probe) beam. The pump pulses are circularly polarized, while the probe pulses are linearly polarized, in order to probe the spin polarization via the magneto-optical Kerr effect. To avoid the build-up of a dynamic nuclear polarization, the helicity of the pump beam is modulated by a liquid crystal modulator and a lock-in scheme is used to increase the detection sensitivity. The two laser beams are colinearly focused via a $100 \times$ microscope objective onto the sample, which is kept in vacuum in a liquid-He cooled cold-finger cryostat at $T=90 \mathrm{~K}$. Fine tuning of the pump-beam path via a piezo-electric mirror holder allows to move the pump spot laterally on the sample surface with respect to the probe spot. Both spots have a diameter of about $1.4 \mu \mathrm{m}$, which enables high-resolution scans. A motorized $x-y$-stage for the cryostat in combination with the piezoelectric mirror holder allows to scan, e.g., the probe beam, while keeping the pump spot position fixed at the sample, or vice versa. The pump beam is filtered out from the backreflected light by a band-pass filter, and the rotation of linear polarization of the probe beam is detected by an optical bridge via two balanced photo diodes.

We start by discussing the experiments on the as-grown wafer. These experiments are similar to the experiments of Ref. 8. Figures 1(c) and 1(d) show lateral line scans of the Kerr signal (probe beam) for different time delays $\Delta t$ between the pump and probe pulses. The circularly polarized pump pulses (spot position fixed at $x, y=0$ ) create a local spin polarization via the excitation of spin-polarized electron-hole pairs due to optical selection rules. Subsequently, the spin polarization is measured via the Kerr rotation of the linearly polarized probe beam. For lateral measurements, the probe spot is moved in $y$ direction on the sample surface with respect to the position of the pump spot [Fig. 1(c)] or in $x$ direction [Fig. 1(d)]. From Figs. 1(c) and 1(d), we can see that the signal at $x, y=0$, i.e., the maximum spin polarization, decreases with increasing $\Delta t$. This is partly due to the recombination of the photo-excited electron-hole pairs and partly due to the diffusion of charge carriers out of the excitation spot with time. For the direction perpendicular to the PSH direction [ $y$ direction, Fig. 1(c)], the lateral Kerr signal exhibits a Gaussian profile, which broadens with increasing $\Delta t$ due to diffusion, while for the direction of the PSH $[x$ direction, Fig. 1(d)] the Kerr signal shows an oscillatory behavior, resembling the PSH. The solid lines in Fig. 1(c) are fits of a Gaussian function, $F\left(y, w_{y}, \Delta t, \tau_{\text {Spin }}\right)=A$ / $\left(w_{y} \sqrt{\pi}\right) \exp \left(-\Delta t / \tau_{\text {Spin }}\right) \exp \left[-\left(y / w_{y}\right)^{2}\right]$, where $A$ is a constant, $w_{y}$ the width of the Gaussian function, $\Delta t$ the time delay between the pump and probe pulses, and $\tau_{\text {Spin }}$ the effective decay time of the spin signal. In Fig. 1(d), the solid lines are fits of a product of a Gaussian and a cosine function, $G\left(x, w_{x}, \Delta t, \tau_{\text {Spin }}, \lambda_{\mathrm{PSH}}\right)=A /\left(w_{x} \sqrt{\pi}\right) \exp (-\Delta t /$ $\left.\tau_{\mathrm{Spin}}\right) \exp \left[-\left(x / w_{x}\right)^{2}\right] \cos \left[\left(2 \pi / \lambda_{\mathrm{PSH}}\right) x\right]$, with the width of the Gaussian function $w_{x}$ and the wavelength of the PSH $\lambda_{\mathrm{PSH}}$. The experimental data points can be fitted quite well by these functions. Figure 2(b) shows $\lambda_{\mathrm{PSH}}$, which is extracted from fits like in Fig. 1(d), for all measured time delays $\Delta t$ between the pump and probe pulses. One can see from this plot that $\lambda_{\text {PSH }}$ starts at a value of about $7.2 \mu \mathrm{m}$ for short time delays and converges towards about $5.4 \mu \mathrm{m}$ with increasing $\Delta t$. It was shown by Salis et $a l .{ }^{20}$ that the finite spatial extension of the initial spin polarization is the reason for this effect and, hence, $\lambda_{\mathrm{PSH}} \sim 5.4 \mu \mathrm{m}$ at long $\Delta t$ is the actual wavelength of the PSH of the system.

After verifying the PSH experimentally in the as-grown sample, which confirms that approximately $\alpha \sim-\beta$ in our sample, we turn now to the investigation of the PSH in 


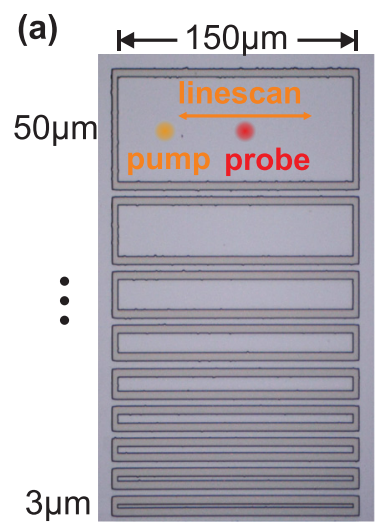

(b)

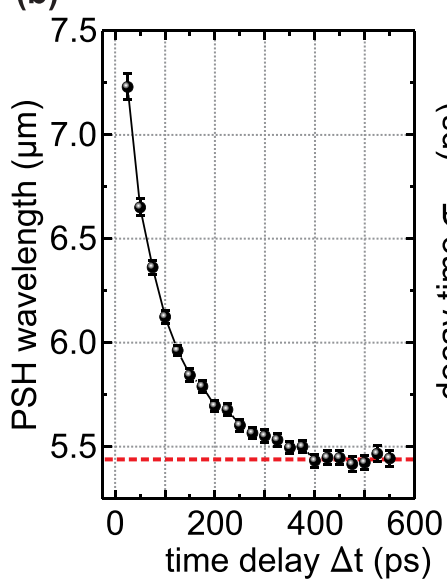

(c)
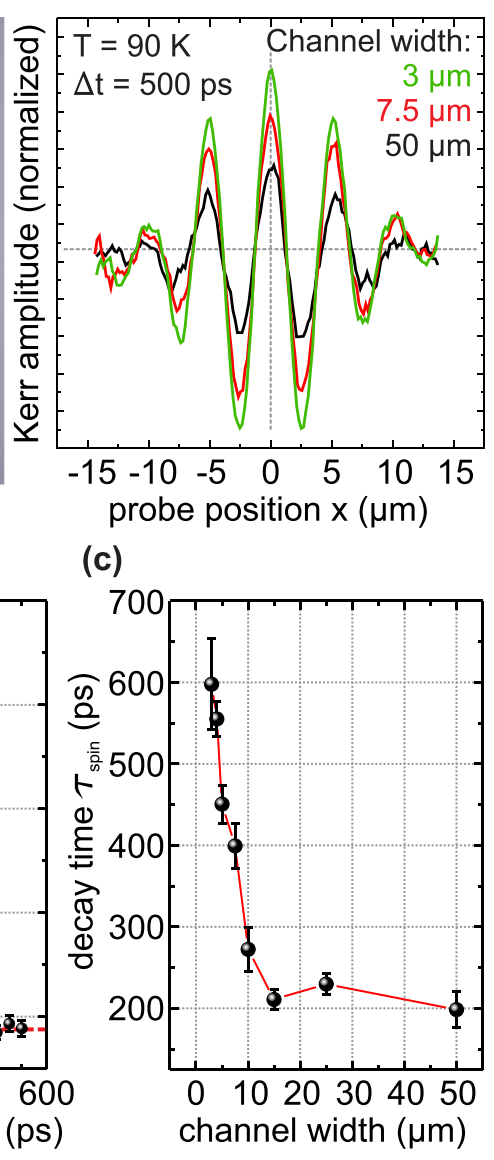

FIG. 2. (a) Microscope image of etched channels along the $x$ direction. The darker gray areas are etched through the quantum well (left panel). Kerr line scans for three selected channel widths (right panel). Each dataset is normalized to its signal strength at $\Delta t=100 \mathrm{ps}$. The line scans were taken, as sketched in the left panel, with fixed probe spot, while the pump spot is scanned. (b) Wavelength of the PSH versus time delay $\Delta t$ between pump and probe pulses. (c) Effective decay time of the spin polarization $\tau_{\text {Spin }}$ versus channel width.

confining structures. The lateral structures are defined via electron-beam lithography and subsequent reactive-ion etching. In the left panel of Fig. 2(a), a microscope image of a processed sample is shown. The darker gray areas are etched through the 2DES. All channels have a length of $150 \mu \mathrm{m}$ and widths between $3 \mu \mathrm{m}$ and $50 \mu \mathrm{m}$. The longitudinal direction of the channels is the $x$ direction, i.e., the direction of the wave vector of the PSH. The right panel of Fig. 2(a) displays the Kerr line scans along $x$ for a fixed time delay of $\Delta t=500$ ps for three selected channel widths of 3, 7.5 and $50 \mu \mathrm{m}$. For each channel width, the signal strength is normalized to the measurement at $\Delta t=100 \mathrm{ps}$ [not shown]. It can be clearly seen from this plot that the signal strength, i.e., the spin polarization, is enhanced with decreasing channel width. This is because the confinement prevents the diffusion of charge carriers in $y$ direction. ${ }^{12}$ In the narrowest channel, the electrons can diffuse essentially in $x$ direction only. Altmann et al. have shown that the lifetime $\tau_{\mathrm{PSH}}$ of the PSH depends only weakly on the confinement and is of order $1 \mathrm{~ns} .{ }^{12}$ They found a transition to 1D diffusion for channels thinner than $8 \mu \mathrm{m}$, employing a model, where the finite injection spot size and the diffusion of electrons out of the excitation spot were taken into account. ${ }^{12}$ For our investigations, we concentrate
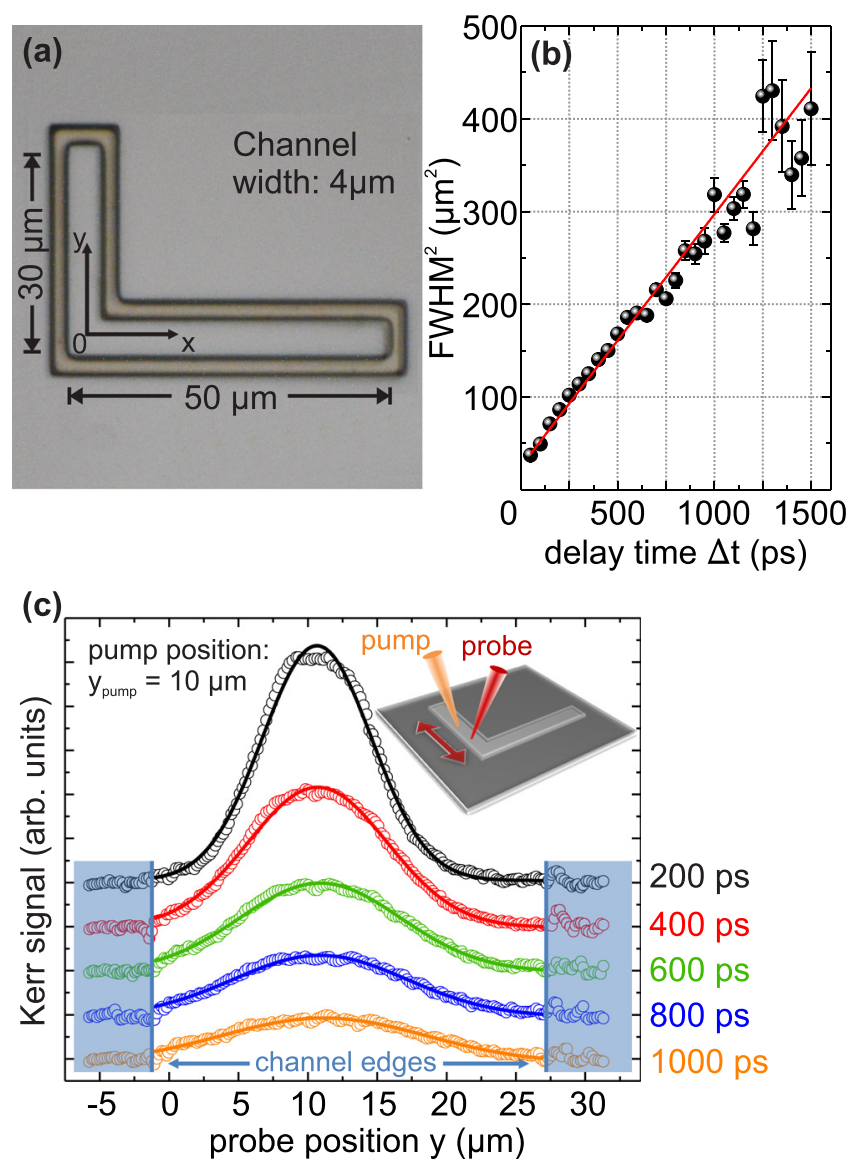

FIG. 3. (a) Microscope image of an L-shaped wire structure. (b) Plot of the squares of the FWHM of measured spin polarization profiles from (c) versus $\Delta t$. The solid line is a linear fit to the data, from which the spin diffusion constant is extracted. (c) Line scans in the $y$ leg of the L-shaped wire structure. The pump position is fixed at $y_{\text {pump }}=10 \mu \mathrm{m}$. The blue-shaded areas mark the regions outside the wire.

on the effective decay time $\tau_{\text {Spin }}$ of the measured spin polarization in the channels. We determine $\tau_{\text {Spin }}$ for different channel widths by taking Kerr scans for different $\Delta t$ and then extract the exponential decay time $\tau_{\text {Spin }}$ of the Kerr signal versus $\Delta t$ from fits, as described above. Figure 2(c) shows the obtained $\tau_{\text {Spin }}$ versus channel width. One can see that $\tau_{\text {Spin }}$ increases by up to a factor of about three in the narrowest channel $(3 \mu \mathrm{m})$ in comparison with the 2D case. Specifically, $\tau_{\text {Spin }}$ increases strongly for channel widths smaller than about $10 \mu \mathrm{m}$, reflecting the transition to $1 \mathrm{D}$ diffusion. ${ }^{12}$

Here, we will demonstrate how we exploit the enhanced effective decay time of spin polarization in the quasi-1D structures to transfer spin polarization in lateral L-shaped wire-like structures to specific spots on the sample. Figure 3(a) shows a microscope image of an L-shaped wire with $4 \mu \mathrm{m}$ channel width. The used coordinate system, with its origin in the corner of the $\mathrm{L}$, is also indicated. The $x$ direction is the direction of the wave vector of the PSH. Figure 3(c) displays Kerr line scans on the $y$ leg of the structure. The blue-shaded areas mark the regions outside the $y$ leg. Comparing these traces with the measurements on the as-grown sample in Fig. 1(c), the impact of the enhanced effective decay time of spin polarization $\tau_{\text {Spin }}$ on the wire structure is obvious: In the wire structure, there is a relatively strong spin signal observable, even for $\Delta t=1000 \mathrm{ps}$, and the measured spin distribution is slightly wider for comparable $\Delta t$, indicating 

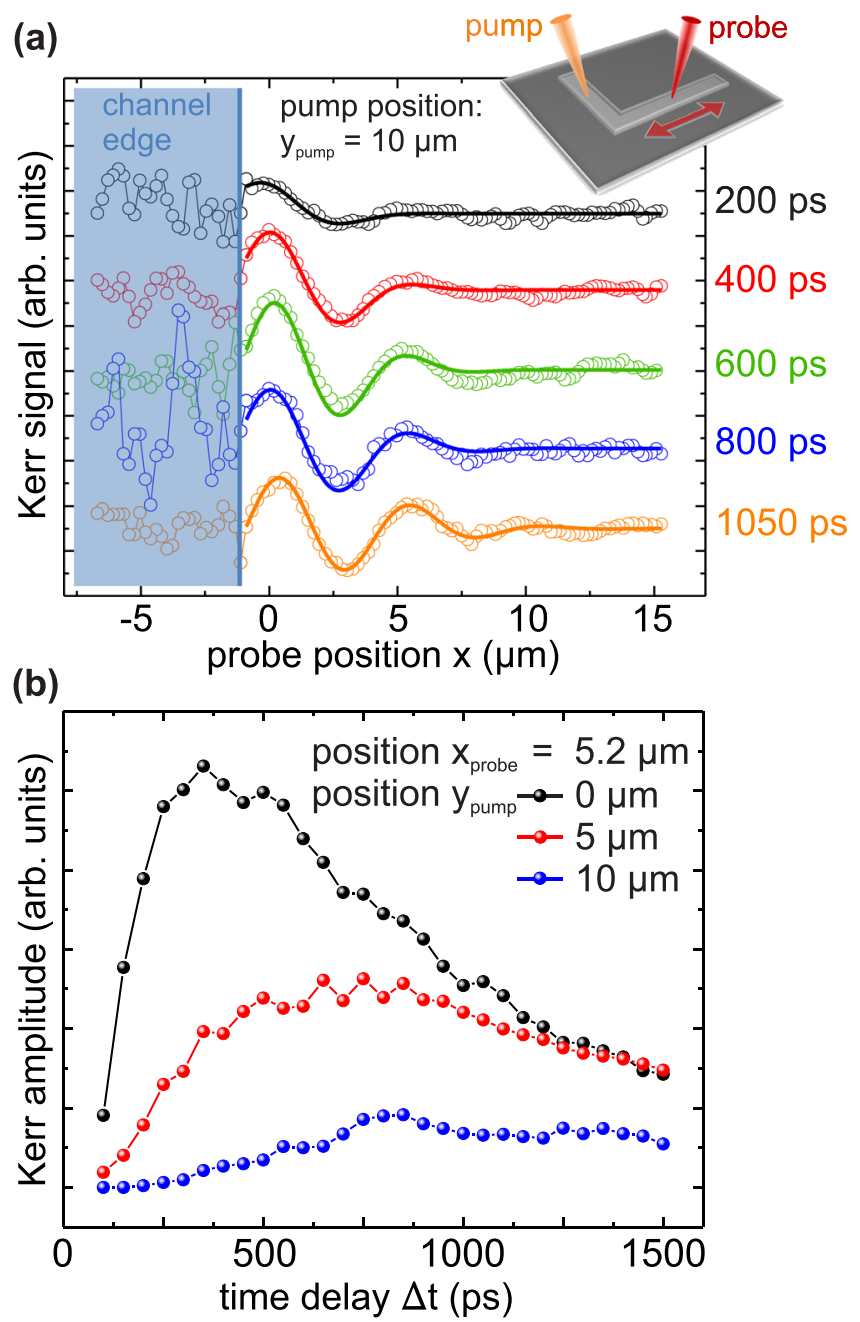

FIG. 4. (a) Line scans in the $x$ leg, while the pump position is fixed at $y_{\text {pump }}=10 \mu \mathrm{m}$ in the $y$ leg. The blue-shaded area marks the region left of the $x$ leg. (b) Kerr amplitude at position $x_{\text {probe }}=5.2 \mu \mathrm{m}$ for different pump positions $y_{\text {pump }}$ versus $\Delta t$.

faster diffusion due to the transition from $2 \mathrm{D}$ to quasi-1D behavior. In Fig. 3(b), the squared FWHM of the measured spin polarization profiles in the $y$ leg are plotted versus $\Delta t$ for all the measured time delays. From the slope of this curve, we extract a spin diffusion constant of about $D_{s}=245 \mathrm{~cm}^{2} / \mathrm{s}$, in agreement with other reports on similar systems. ${ }^{12,21}$ In the measurements displayed in Fig. 4(a), the pump spot is fixed at position $y_{\text {pump }}=10 \mu \mathrm{m}$ in the $y$ leg, while the probe spot is scanned in the $x$ leg for different $\Delta t$. Here, the blue-shaded area marks the outside region left of the $x$ leg [cf. Fig. 3(a)]. From these measurements, one can read that, from spin-up initialization at position $y_{\text {pump }}=10 \mu \mathrm{m}$, a spin down polarization is, e.g., transferred to position $x \sim 2.6 \mu \mathrm{m}$, while spin up is at position $x \sim 5.2 \mu \mathrm{m}$. Furthermore, we find from these investigations that spin polarization can be transferred in the wire-like structures around a corner over a total distance of at least $20 \mu \mathrm{m}$, while in the $2 \mathrm{D}$ structure the spin signal has vanished already after a distance of about $10 \mu \mathrm{m}$ (cf. Fig. 1(d)). Figure 4(b) shows the Kerr amplitude in the $x$ leg at position $x_{\text {probe }}=5.2 \mu \mathrm{m}$ versus time delay for different pump positions $y_{\text {pump }}$ in the $y$ leg. By increasing the distance of the pump position in $y$ direction, one can see here the build-up of the spin signal in the $x$ leg.

In conclusion, we have demonstrated a strongly enhanced effective decay time of the optically induced spin polarization in wire-like structures, based on a modulation-doped GaAs quantum well with a 2DES in the regime of the PSH. Via L-shaped lateral structures, we could show that the spin polarization can be deterministically transferred over distances of at least $20 \mu \mathrm{m}$, even around a corner.

We acknowledge the financial support by the Deutsche Forschungsgemeinschaft via SFB 689 and project SCHU1171/7.

${ }^{1}$ S. Datta and B. Das, Appl. Phys. Lett. 56, 665 (1990).

${ }^{2}$ B. A. Bernevig, J. Orenstein, and S.-C. Zhang, Phys. Rev. Lett. 97, 236601 (2006).

${ }^{3}$ Y. A. Bychkov and E. I. Rashba, Pis'ma Z. Eksp. Teor. Fiz. 39, 66 (1984) [JETP Lett. 39, 78 (1984)].

${ }^{4}$ G. Dresselhaus, Phys. Rev. 100, 580 (1955).

${ }^{5}$ M. I. D'yakonov and V. I. Perel', Z. Éksp. Teor. Fiz. 60, 1954 (1971) [Sov. Phys. JEPT 33, 1053 (1971)].

${ }^{6}$ J. Schliemann, J. C. Egues, and D. Loss, Phys. Rev. Lett. 90, 146801 (2003).

${ }^{7}$ J. D. Koralek, C. P. Weber, J. Orenstein, B. A. Bernevig, S.-C. Zhang, S. Mack, and D. D. Awshalom, Nature 458, 610 (2009).

${ }^{8}$ M. P. Walser, C. Reichl, W. Wegscheider, and G. Salis, Nat. Phys. 8, 757 (2012).

${ }^{9}$ M. Kohda, V. Lechner, Y. Kunihashi, T. Dollinger, P. Olbrich, C. Schönhuber, I. Caspers, V. V. Bel'kov, L. E. Golub, D. Weiss, K. Richter, J. Nitta, and S. D. Ganichev, Phys. Rev. B 86, 081306 (2012).

${ }^{10}$ J. Wunderlich, B. G. Park, A. C. Irvine, L. P. Zarbo, E. Rozkotova, P. Nemec, V. Novak, J. Sinova, and T. Jungwirth, Science 330, 1801 (2010).

${ }^{11}$ J. Ishihara, Y. Ohno, and H. Ohno, Phys. Exp. 7, 013001 (2014).

${ }^{12}$ P. Altmann, M. P. Walser, C. Reichl, W. Wegscheider, and G. Salis, Phys. Rev. B 90, 201306(R) (2014).

${ }^{13}$ L. Yang, J. D. Koralek, J. Orenstein, T. R. Tibbetts, J. L. Reno, and M. P. Lilly, Nat. Phys. 8, 153 (2012).

${ }^{14}$ L. Yang, J. D. Koralek, J. Orenstein, T. R. Tibbetts, J. L. Reno, and M. P. Lilly, Phys. Rev. Lett. 109, 246603 (2012).

${ }^{15}$ C. Schönhuber, M. P. Walser, G. Salis, C. Reichl, W. Wegscheider, T. Korn, and C. Schüller, Phys. Rev. B 89, 085406 (2014).

${ }^{16}$ P. Altmann, F. G. G. Hernandez, G. J. Ferreira, M. Kohda, C. Reichl, W. Wegscheider, and G. Salis, Phys. Rev. Lett. 116, 196802 (2016).

${ }^{17}$ Y. Kunihashi, H. Sanada, H. Gotoh, K. Onomitsu, M. Kohda, J. Nitta, and T. Sogawa, Nat. Commun. 7, 10722 (2016).

${ }^{18}$ M. Studer, G. Salis, K. Ensslin, D. C. Driscoll, and A. C. Gossard, Phys. Rev. Lett. 103, 027201 (2009).

${ }^{19}$ The photoluminescence spectrum of the quantum well (not shown) has a maximum at about $807 \mathrm{~nm}$ and a high-energy cutoff, reflecting the Fermi energy, where the absorptive region starts, at around $795 \mathrm{~nm}$.

${ }^{20}$ G. Salis, M. P. Walser, P. Altmann, C. Reichl, and W. Wegscheider, Phys. Rev. B 89, 045304 (2014).

${ }^{21}$ R. Völkl, M. Schwemmer, M. Griesbeck, S. A. Tarasenko, D. Schuh, W. Wegscheider, C. Schüller, and T. Korn, Phys. Rev. B 89, 075424 (2014). 